\title{
Iterative Learning Control for Variable Pass Length Systems
}

\author{
Thomas Seel* Thomas Schauer* Jörg Raisch*,** \\ * Control Systems Group, Technische Universität Berlin, Germany \\ (e-mail: seel@control.tu-berlin.de). \\ ** Systems and Control Theory Group, Max Planck Institute for \\ Dynamics of Complex Technical Systems, Magdeburg, Germany
}

\begin{abstract}
Monotonic convergence of linear iterative learning control (ILC) systems with changing pass length is considered. The maximum pass length (MPL) error is introduced as a useful concept for convergence analysis of this class of systems. Using the lifted-system framework, a both necessary and sufficient monotonic convergence criterion is found for the 1 -norm of the MPL error. Further findings on 2-norm and $\infty$-norm convergence are added. Finally, an example system is given, i.e. the control of an electrical stimulation system for gait assistance, and simulation results are provided.
\end{abstract}

Keywords: iterative learning control, variable pass length, discrete-time, linear systems, lifted system framework, convergence analysis, monotonic convergence, biomedical systems

\section{INTRODUCTION}

Iterative learning control makes use of the fact that the performance of a system that executes the same task multiple times can be improved by learning from previous executions (trials, iterations, passes) [1]. Therefore it was found to have great potential for applications with a naturally repetitive action [2]. However, for ILC theory to be applicable, certain assumptions must hold:

- the dynamics do not change from pass to pass, i.e. the same input causes the same output ${ }^{1}$

- no input saturation shall occur

- the pass length/trial duration does not change

If this can be assured, then a well-developed theoretical framework can be applied that is briefly described (for the linear case) in the following.

\subsection{Linear ILC with constant pass length}

Due to the need of data storage, discrete-time is considered the natural domain for ILC analysis [1]. For a causal singleinput single-output system with relative degree $m$, let

$$
\begin{aligned}
u_{j} & =\left[u_{j}(1-m), u_{j}(2-m), \ldots, u_{j}(n-m)\right]^{T} \in \mathbb{R}^{n}, \\
y_{j} & =\left[y_{j}(1), y_{j}(2), \ldots, y_{j}(n)\right]^{T} \in \mathbb{R}^{n}, \\
v & =[v(1), v(2), \ldots, v(n)]^{T} \in \mathbb{R}^{n}
\end{aligned}
$$

be the lifted signal vectors (i.e. the vectors of a finite number of sequent sample values) of the input signal, of the output signal, and of an unknown, but iteration-invariant, disturbance signal, respectively. Furthermore, $n \in \mathbb{N}$ is the pass length and $j \in \mathbb{N}_{0}$ is the iteration/trial/pass index. Then the system dynamics shall be given by

$$
y_{j}=P u_{j}+v \text {, }
$$

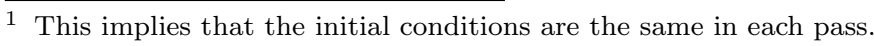

where $P \in \mathbb{R}^{n \times n}$ is a, in general regular, lower triangular matrix called the lifted system matrix of the process. (For a short example, please see Appendix A.) Furthermore, the error $e_{j}=\left(y_{d}-y_{j}\right) \in \mathbb{R}^{n}$ is defined with respect to a desired output $y_{d} \in \mathbb{R}^{n}$, and the control task is to successively, i.e. from pass to pass, reduce the error (in some norm) to a small number. This shall be achieved using the following input update law

$$
u_{j+1}=Q\left(u_{j}+L e_{j}\right) \text {, }
$$

where $Q, L \in \mathbb{R}^{n \times n}$ need to be designed accordingly. Together with (1) this results in the following error dynamics $e_{j+1}=P Q P^{-1}\left(I_{n}-P L\right) e_{j}+\left(I_{n}-P Q P^{-1}\right)\left(y_{d}-v\right)$. From that it is concluded that the error converges to a finite value ${ }^{2} e_{\infty}:=\lim _{j \rightarrow \infty} e_{j}$ if and only if the spectral radius $\rho\left(P Q P^{-1}\left(I_{n}-P L\right)\right)=\rho\left(Q\left(I_{n}-L P\right)\right)$ is smaller than one [3]. But this does not eliminate the danger of large transient growth. Thus instead, it is often demanded that the largest singular value $\bar{\sigma}\left(P Q P^{-1}\left(I_{n}-P L\right)\right)$ is smaller than unity, which guarantees (strict) monotonic convergence (MC) of the Euclidean norm $\left\|e_{j}-e_{\infty}\right\|_{2}[1]$. For unknown $v$, this condition is also necessary, which can be seen, e.g., by choosing $v$ such that $e_{0}=\left(y_{d}-P u_{0}-v\right)$ points into the direction of maximum gain of $\left(P Q P^{-1}\left(I_{n}-\right.\right.$ $P L)$ ). Also, please note that the MC condition might be rewritten in any other vector norm and its induced matrix norm.

\subsection{Applications and shortcomings}

The outlined algorithm has been modified in many ways and has been successfully used in a multitude of application systems including robotics, process control, and biological systems (e.g. [6], [4], and many more). Especially for biomedical systems, learning-type control is

$\overline{2}$ Precisely, $e_{\infty}=\left(I_{n}-P Q P^{-1}\left(I_{n}-P L\right)\right)^{-1}\left(I_{n}-P Q P^{-1}\right)\left(y_{d}-v\right)$ 
believed to have a dramatic progress in the near future [5]. Besides using the introduced lifted-system framework, controller design in the frequency domain is common for many applications, especially in robotics. Useful additional properties $^{3}$ are found in the $\mathrm{z}$-domain [1], but for this technique to be applicable, the trial duration must be magnitudes larger than both the sampling time and the system's characteristic time. Apparently, this is not the case for many ILC applications in biomedical engineering. In contrast, it is rather often the case that not even the assumptions made at the beginning of this section can be assured. Two examples concerning functional electrical stimulation (FES) shall be presented briefly here:

FES for upper limb movement: A complex functional arm movement shall be produced via electrical stimulation of the respective muscles through skin electrodes. For this purpose, very precise stimulation patterns need to be applied. However, the system dynamics are hardly known and depend on many details such as the exact electrode position. For successive learning of the stimulation profile, the use of a linear ILC algorithm seems promising. However, at least for the first passes, it is expected that the arm position leaves the neighborhood of the reference trajectory very early. The data gathered outside this neighborhood is hardly useful since the dynamics and disturbances vary over state space. Therefore, and for reasons of safety, a trial needs to be terminated whenever output and reference begin to differ too much. Nevertheless, some valuable data is gathered that might be used for learning if there was a way to deal with varying pass lengths.

FES for gait assistance: Stroke patients walking on a treadmill can be supported, for example, by controlling the ankle joint angle via electrical stimulation of the tibialis anterior muscle. Due to the repetitive nature of gait, ILC was successfully used to learn the stimulation profile for the swing phase within a small number of steps, see [9]. However, the hip joint and the knee joint are actuated by the patients themselves. Thus, depending on their strength and abilities, the steps are usually cut short by suddenly putting the foot down. Assuming that up to this point the movement of hip and knee was hardly different from the movement in a full-length step, the data gathered in these aborted steps should be used for learning.

Both example applications demonstrate that in many situations a pass might be terminated early, either by events that depend on the states of the system or on the controller performance, or by randomly occurring events. Typically, this problem is either ignored completely, or a heuristic approach is used hoping for convergence to be maintained (see e.g. [9], [8]). But currently there is no ILC theory that guarantees stability or monotonic convergence for such variable pass length systems. In this publication, first results are presented concerning this problem, and their usefulness for practice is discussed.

\section{VARIABLE PASS LENGTH SYSTEMS}

A new class of iterative learning systems is introduced and its convergence properties are analyzed. It is assumed, that the pass length $n$ can vary from pass to pass and that not

\footnotetext{
3 For example, the z-domain counterparts of $P, L, Q$ commute.
}

a single element of the sequence of pass lengths $\left\{n_{j}\right\}$ is known in advance. However, a possibly very small lower bound $\underline{n}$ and a possibly very large upper bound $\bar{n}$ are given. Consequently, a full-length desired output $y_{d} \in \mathbb{R}^{\bar{n}}$ must be defined, and for each pass a full-length input $u \in \mathbb{R}^{\bar{n}}$ must be available. In order to cope with the varying length of the output vector and for the sake of a clearer notation, the remove-last- $\left(\bar{n}-n_{j}\right)$-rows operator $[\cdot]_{n_{j}}: \mathbb{R}^{\bar{n}} \rightarrow \mathbb{R}^{n_{j}}$ and the append- $\left(\bar{n}-n_{j}\right)$-zero-rows operator $[\cdot]_{\bar{n}}: \mathbb{R}^{n_{j}} \rightarrow \mathbb{R}^{\bar{n}}$ are introduced ${ }^{4}$. Thus, e.g., the desired output signal for pass length $n_{j}$ becomes $\left[y_{d}\right]_{n_{j}}$.

\subsection{Linear System Dynamics}

Consider a causal linear iterative process with bounded input $\hat{u}_{j} \in \mathbb{R}^{n_{j}}$, output $\hat{y}_{j} \in \mathbb{R}^{n_{j}}$ and variable pass length $n_{j} \in[\underline{n}, \bar{n}]$. The dynamics of the process can be written in the lifted-system framework as follows

$$
\hat{y}_{j}=\left[y_{j}\right]_{n_{j}}=\left[P u_{j}+v\right]_{n_{j}},
$$

where $u_{j} \in \mathbb{R}^{\bar{n}}$ and $v \in \mathbb{R}^{\bar{n}}$ are the (bounded) input and the disturbance, respectively, that would act on the system if $n_{j}$ was equal to $\bar{n}$. Accordingly, $y_{j} \in \mathbb{R}^{\bar{n}}$ is the output for maximum pass length (MPL), and the MPL error $e_{j} \in \mathbb{R}^{\bar{n}}$ can be defined as

$$
e_{j}=y_{d}-y_{j}
$$

Apparently, both the MPL output $y_{j}$ and the MPL error $e_{j}$ are only theoretical concepts and not measurable signals. In practice, the $j$-th pass actually terminates after $n_{j}$ samples. Thus, only $\hat{y}_{j}$ (but not $y_{j}$ ) can be measured and used for learning. A fairly general input update law is

$$
\begin{aligned}
u_{j+1} & =Q\left(u_{j}+L\left[\hat{y}_{j}-\left[y_{d}\right]_{n_{j}}\right]_{\bar{n}}\right) \\
& =Q\left(u_{j}+L H_{n_{j}} e_{j}\right),
\end{aligned}
$$

where $Q, L \in \mathbb{R}^{\bar{n} \times \bar{n}}$ are the Q-filter and the learning gain matrix, respectively, and the last $\left(\bar{n}-n_{j}\right)$ samples of $e_{j}$ are set to zero by multiplication by the block-diagonal matrix ${ }^{5} H_{n_{j}}=\operatorname{blockdiag}\left\{I_{n_{j}}, 0_{\bar{n}-n_{j}}\right\}$, with $I_{n_{j}}$ and $0_{\bar{n}-n_{j}}$ being identity and zero square matrices, respectively.

\subsection{Convergence Analysis}

Although $e_{j}$ is not a measurable signal, its convergence properties well describe whether the controller performance actually improves. In contrast, the amount of information in the measured error $\left(\left[y_{d}\right]_{n_{j}}-\hat{y}_{j}\right)$ strongly depends on the current pass length $n_{j}$. Furthermore, please note that if $\left\|e_{j}\right\|_{p}$ is monotonically decreasing in $j$, then also $\left\|\left[y_{d}\right]_{n_{j}}-\hat{y}_{j}\right\|_{p}$ is bounded by decreasing upper bounds, e.g. $\left\|\left[y_{d}\right]_{n_{j}}-\hat{y}_{j}\right\|_{p} \leq\left\|e_{j}\right\|_{p} \forall j \forall p \in\{1,2, \infty\}$. Therefore, the concept of the MPL error is crucial for convergence analysis. For a regular system matrix $P$, the MPL error dynamics are obtained by combining (3), (4), and (5):

$$
\begin{aligned}
e_{j+1} & =P Q P^{-1}\left(I_{\bar{n}}-P L H_{n_{j}}\right) e_{j} \\
& +\left(I_{\bar{n}}-P Q P^{-1}\right)\left(y_{d}-v\right)
\end{aligned}
$$

The special case of the Q-filter being identity is considered, i.e. $Q=I_{\bar{n}}$. (Note that in this case the second summand in (6) is zero.) In order to have monotonic convergence

\footnotetext{
4 Both operators may also be written as multiplication by a matrix consisting of the unit matrix $I_{n_{j}}$ and $\left(\bar{n}-n_{j}\right)$ zero columns/rows.

5 By definition, $H_{n_{j}} a=\left[[a]_{n_{j}}\right]_{\bar{n}} \forall a \in \mathbb{R}^{\bar{n}}$
} 
(MC) for an arbitrary sequence of pass lengths $\left\{n_{j}\right\}$ and an arbitrary disturbance signal $v$, the following condition must hold:

Lemma 1. (MC for variable pass lengths). For a repetitive process (3) with regular system matrix $P$ and input update law (5) with Q-Filter $Q=I_{\bar{n}}$, the monotonic convergence condition of the MPL error is

$$
\begin{aligned}
\left\|e_{j+1}\right\|_{p} & \leq\left\|e_{j}\right\|_{p} & & \forall j,\left\{n_{j}\right\}, v \\
\Leftrightarrow\left\|I_{\bar{n}}-P L H_{n}\right\|_{p} & \leq 1 & & \forall n \in[\underline{n}, \bar{n}]
\end{aligned}
$$

for some vector norm $\|\cdot\|_{p}$ and its induced matrix norm.

In practice, this would entail the need to calculate the norm of $(\bar{n}-\underline{n}+1)$ matrices of dimension $\bar{n} \times \bar{n}$, which is a large number of large-scale matrices when high sampling rates are used. Fortunately, simplified conditions can be found for the 1 -norm $\left\|e_{j}\right\|_{1}$ and the $\infty$-norm ${ }^{6}\left\|e_{j}\right\|_{\infty}$. To find and prove the below theorems, the special structure of the matrices $\left(I_{\bar{n}}-P L H_{n}\right)$ is exploited. An example is provided in Appendix B.

Theorem 1. (MC in 1-norm). Given a repetitive process (3) with regular $P$, input update law (5), and with $Q=$ $I_{\bar{n}}$, the 1 -norm of the MPL error $e_{j}$ is monotonically decreasing, i.e.

if and only if

$$
\left\|e_{j+1}\right\|_{1} \leq\left\|e_{j}\right\|_{1} \forall j,\left\{n_{j}\right\}, v,
$$

$$
\gamma:=\left\|I_{\bar{n}}-P L\right\|_{1} \leq 1
$$

Proof. Necessity follows from Lemma 1, as (7) repeats a special case $(n=\bar{n}, p=1)$ of the condition therein. Sufficiency is proved by the following argument, which holds for every $n \in[\underline{n}, \bar{n})$ :

$$
\left(I_{\bar{n}}-P L H_{n}\right)=\left(I_{\bar{n}}-P L\right) H_{n}+\left(I_{\bar{n}}-H_{n}\right)
$$

Note that the last $\bar{n}-n$ columns of $\left(I_{\bar{n}}-P L\right) H_{n}$ are zero and that $\left(I_{\bar{n}}-H_{n}\right)=\operatorname{blockdiag}\left\{0_{n}, I_{\bar{n}-n}\right\}$. Thus, for the maximum absolute column sum norm $\|\cdot\|_{1}$, we have

$\left\|I_{\bar{n}}-P L H_{n}\right\|_{1}=\max \left(\left\|\left(I_{\bar{n}}-P L\right) H_{n}\right\|_{1}, 1\right) \leq \max (\gamma, 1)$. And with Lemma 1, monotonic convergence follows.

Of course, strict monotonic convergence is desirable. But even for $\gamma<1$, it turns out that $\left\|I_{\bar{n}}-P L H_{n}\right\|_{1}=1 \forall n<\bar{n}$ since the last $\bar{n}-n$ columns each have absolute sum of one. To clarify this issue, the following corollary is given:

Corollary 1. If the matrix $L$ satisfies $\gamma=\left\|I_{\bar{n}}-P L\right\|_{1}<1$, then $\left\|e_{j+1}\right\|_{1}<\left\|e_{j}\right\|_{1}$ for arbitrary pass length $n_{j} \in[\underline{n}, \bar{n}]$, unless the first $n_{j}$ entries of $e_{j}$ are zero. This follows from

$$
\left\|e_{j+1}\right\|_{1} \leq\left\|\left(I_{\bar{n}}-P L\right)\right\|_{1} \cdot \sum_{k=1}^{n_{j}}\left|\left(e_{j}\right)_{k}\right|+\sum_{k=n_{j}+1}^{\bar{n}}\left|\left(e_{j}\right)_{k}\right|
$$

where $k$ denotes the row index of the MPL error vector.

In practice, that means that the algorithm cannot improve the last input samples until long enough passes occur. But it always learns from the first samples and improves controller performance, even for short pass lengths.

Theorem 2. (MC in $\infty$-norm). For a repetitive process (3) with regular $P$, input update law (5), and with $Q=I_{\bar{n}}$, the $\infty$-norm of the MPL error is MC, i.e.

$$
\left\|e_{j+1}\right\|_{\infty} \leq\left\|e_{j}\right\|_{\infty} \forall j,\left\{n_{j}\right\}, v,
$$

$\overline{{ }^{6}\|x\|_{1}=\sum_{1}^{n}|x(n)| \forall x \in \mathbb{R}^{n} \text { and }\|x\|_{\infty}}=\max _{n}|x(n)| \forall x \in \mathbb{R}^{n}$ if and only if

$$
\left\|I_{\bar{n}}-P L\right\|_{\infty} \leq 1 \wedge(P L)_{i, k}=0 \forall k<i \forall i>\underline{n},
$$

where $i$ and $k$ are the row and column index, respectively.

Proof. Sufficiency and necessity is proved by the following argument, which holds for every $n \in[\underline{n}, \bar{n})$ :

$$
\begin{aligned}
\left(I_{\bar{n}}-P L H_{n}\right) & =H_{n}\left(I_{\bar{n}}-P L\right) H_{n} \\
& -\left(I_{\bar{n}}-H_{n}\right) P L H_{n}+\left(I_{\bar{n}}-H_{n}\right),
\end{aligned}
$$

as $H_{n} H_{n}=H_{n}$. Note that the last $\bar{n}-n$ rows (and columns) of $H_{n}\left(I_{\bar{n}}-P L\right) H_{n}$ are zero, that the first $n$ rows and the last $\bar{n}-n$ columns of $\left(I_{\bar{n}}-H_{n}\right) P L H_{n}$ are zero, and that $\left(I_{\bar{n}}-H_{n}\right)=\operatorname{blockdiag}\left\{0_{n}, I_{\bar{n}-n}\right\}$. From that it follows for the maximum absolute row sum norm $\|\cdot\|_{\infty}$ :

$$
\begin{array}{r}
\left\|I_{\bar{n}}-P L H_{n}\right\|_{\infty}=\max \left(\left\|H_{n}\left(I_{\bar{n}}-P L\right) H_{n}\right\|_{\infty},\right. \\
\left.\max _{i>n} \sum_{k=1}^{n}\left|(P L)_{i, k}\right|+1\right)
\end{array}
$$

Note that the right-hand side is less than or equal to one for all $n \in[\underline{n}, \bar{n}]$ if and only if (9) holds. Combining this with Lemma 1 completes the proof.

As for the 1-norm, we find that even if (9) holds with $\left\|I_{\bar{n}}-P L\right\|_{\infty}$ being strictly less than unity, we still have $\left\|I_{\bar{n}}-P L H_{n}\right\|_{\infty}=1$ for all pass lengths $n<\bar{n}$. However, as a corollary of the above proof, we find for the MPL error:

$$
\begin{array}{r}
\left\|e_{j+1}\right\|_{\infty} \leq \max \left(|| H_{n_{j}}\left(I_{\bar{n}}-P L\right) H_{n_{j}} \|_{\infty} \cdot \max _{k \in\left[1, n_{j}\right]}\left|\left(e_{j}\right)_{k}\right|,\right. \\
\left.\max _{k \in\left(n_{j}, \bar{n}\right]}\left|\left(e_{j}\right)_{k}\right|\right) \leq \max _{k \in[1, \bar{n}]}\left|\left(e_{j}\right)_{k}\right|=|| e_{j} \|_{\infty}
\end{array}
$$

And since $\left\|H_{n_{j}}\left(I_{\bar{n}}-P L\right) H_{n_{j}}\right\|_{\infty} \leq\left\|I_{\bar{n}}-P L\right\|_{\infty}$, it is found that $\left((9) \wedge\left\|I_{\bar{n}}-P L\right\|_{\infty}<1\right) \Rightarrow\left\|e_{j+1}\right\|_{\infty}<\left\|e_{j}\right\|_{\infty}$, unless (at least one of) the sample(s) with the largest absolute error, i.e. $\left\|e_{j}\right\|_{\infty}$, is among the last $\left(\bar{n}-n_{j}\right)$ samples. In practice, this means that the controller performance improves (in the sense of the $\infty$-norm of the MPL error) in each pass that is long enough to contain the sample(s) of largest error.

Please note that both Theorems 1 and 2 imply that only the maximum pass length matrix $\left(I_{\bar{n}}-P L\right)$ and its norm need to be calculated to check for monotonic convergence. But in the $\infty$-norm case, perfect plant inversion ${ }^{7}$ is required, at least in the lower left corner of $(P L)$. Therefore this criterion is deemed less practical.

Convergence of the 2-norm: No criterion is found for MC of $\left\|e_{j}\right\|_{2}$ that significantly simplifies the condition given by Lemma 1. But based on the above criteria for MC of the MPL error in the 1 -norm and the $\infty$-norm, the dynamic behavior of $\left\|e_{j}\right\|_{2}$ can be assessed using the well known facts that $\|A\|_{2} \leq\|A\|_{1} \cdot\|A\|_{\infty} \forall A \in \mathbb{R}^{n \times n}$ and that $\|a\|_{\infty} \leq\|a\|_{2} \leq\|a\|_{1} \forall a \in \mathbb{R}^{n}$. Precisely, the following properties shall be noted:

- If the MPL error is MC both in the 1-norm and in the $\infty$-norm, then it is also MC in the 2-norm.

- If the MPL error converges to zero in any of the three norms, then so it does in the other two.

- If its 1-norm converges to a small value $\varepsilon$ then both its 2 -norm and its $\infty$-norm converge to or even fall below $\varepsilon$, with the 1-norm as an upper bound on both.

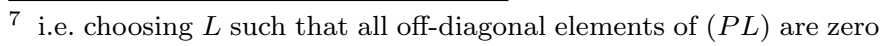


Therefore, it is in general sufficient to design a learning gain matrix $L$ that satisfies the condition (7).

Design of a Q-Filter: In Lemma 1 and in Theorems 1 and 2 , the Q-filter has been omitted by choosing $Q=I_{\bar{n}}$. This is a reasonable choice since it sets the second summand in (6) to zero, and therefore reduces steady-state errors. If a low-pass filter is used instead, which is a common choice in many applications [1], then convergence cannot be guaranteed for all sequences of pass lengths $\left\{n_{j}\right\}$ and disturbances $v$. Even the deceptively intuitive choice of a Q-Filter that assures $\left\|P Q P^{-1}\left(I_{\bar{n}}-P L H_{n}\right)\right\|_{p} \leq 1$ (cf. (6)) does not necessarily provide this property. However, simulation results, e.g. those in Section 3, indicate that in many cases monotonic convergence can be established, at least for a wide range of $\left\{n_{j}\right\}$ and $v$. Therefore further research is needed on necessary and sufficient MC conditions in the case of Q-filtering.

\section{CONTROLLER DESIGN AND SIMULATION RESULTS FOR AN EXAMPLE SYSTEM}

The goal of this section is to illustrate some variable pass length ILC effects and to demonstrate the usefulness of the criteria found above. For this purpose, controller design and simulations are carried out for a simple example application system that is motivated by a recent publication by Nahrstaedt et al. [9]. Therein, functional electrical stimulation of the tibialis anterior muscle is used to produce a predefined foot movement during the swing phase of gait (i.e. when the foot moves forward).

\subsection{FES System Model}

The stimulation intensity, i.e. the pulse width in tenths of milliseconds, and the ankle joint angle in degrees shall be the input variable $u$ and the output variable $y$, respectively. Then the following linear difference equation model approximates the system dynamics at a sampling time $T_{s}=0.02 \mathrm{~s}$ (cf. [10]):

$$
\begin{aligned}
& y(t)+a_{1} y\left(t-T_{s}\right)+a_{2} y\left(t-2 T_{s}\right)=b u\left(t-2 T_{s}\right), \\
& a_{1}=-0,8097002, a_{2}=-0.0777289, b=0.6634
\end{aligned}
$$

Based on experimental data, the duration of swing phase in the gait of stroke patients is estimated to vary between $0.6 \mathrm{~s}$ and $1.0 \mathrm{~s}$, which corresponds to $\underline{n}=30$ and $\bar{n}=50$. Therefore, the following lifted signal vectors are defined:

$$
\begin{aligned}
u_{j} & =\left[u_{j}\left(t_{0}-T_{s}\right), u_{j}\left(t_{0}\right), \ldots, u_{j}\left(t_{0}+48 T_{s}\right)\right]^{T} \\
y_{j} & =\left[y_{j}\left(t_{0}+T_{s}\right), y_{j}\left(t_{0}+2 T_{s}\right), \ldots, y_{j}\left(t_{0}+50 T_{s}\right)\right]^{T} \\
v & =\left[v\left(t_{0}+T_{s}\right), v\left(t_{0}+2 T_{s}\right), \ldots, v\left(t_{0}+50 T_{s}\right)\right]^{T}
\end{aligned}
$$

Accordingly, the (lower triangular Toeplitz) lifted system matrix $P$ is calculated. Based on experimental data, reasonable values for the disturbance signal $v$ and the desired output $y_{d}$ are generated, and the initial input $u_{0}$ is chosen to be a constant pulse width of $0.2 \mathrm{~ms}$. (Please refer to Appendix $\mathrm{C}$ for the numerical values of $P, v$, and $y_{d}$.)

\subsection{Variable Pass Length ILC Design}

Each input $u_{j}, j>0$ is calculated using the input update law (5). For controller design, two strategies are employed:
- a diagonal learning gain matrix $L_{1}$, with $L_{1}(i, i)=l_{0}$ on the main diagonal

- a two-parameter learning gain matrix $L_{2}$, with $L_{2}(i, i)=l_{1}$ on the main diagonal, and $L_{2}(i+1, i)=l_{2}$ just below the diagonal

In the following, both approaches are employed without and in combination with a Q-filter, while the convergence properties of the MPL error are analyzed using the findings in Section 2.2. During this design process three characteristic cases of convergence quality are encountered successively:

The worst case: At first, the matrix $L_{1}$ is designed by gradually increasing $l_{0}$ (starting from zero) until overshoot $^{8}$ effects start to appear in the simulated outputs $y_{j}, j \in[0,6]$. Although the resulting gain $l_{0}=0.12$ gives $\left\|I_{\bar{n}}-P L_{1}\right\|_{2}<1$ (i.e. the criterion used in [10]), it is found by calculation that this is not true for shorter passes, and that $\left\|I_{\bar{n}}-P L_{1} H_{n}\right\|_{p}>1.5 \forall p \in\{1, \infty\} \forall n \in[\underline{n}, \bar{n}]$. With Lemma 1 it follows that (although it does for the values defined above) $L_{1}$ does not do provide MC for all possible disturbance signals $v$ and sequences $\left\{n_{j}\right\}$. (Precisely, that is true for all diagonal gains $l_{0} \neq 0$.) Therefore this represents the worst case where for safe use in practice either additional knowledge on the actual $v$ or $\left\{n_{j}\right\}$ is needed, or another controller structure must be used.

The best case: Next, the matrix $L_{2}$ is designed by numerically minimizing $\left\|I_{\bar{n}}-P L_{2}\right\|_{1}$ over wide ranges of both $l_{1}$ and $l_{2}$. The use of a second parameter proves to be very advantageous. For $l_{1}=1.5, l_{2}=-1.35$ an approximate plant inversion with a small MPL matrix 1norm of $\gamma<0.13$ is gained. Therefore strict monotonic convergence of $\left\|e_{j}\right\|_{1}$ (for all $j,\left\{n_{j}\right\}, v$ ) follows from Corollary 1 without further computational effort. By calculation of $\max _{n}\left\|I_{\bar{n}}-P L_{2} H_{n}\right\|_{2} \approx 1.004$ it is, moreover, found that $\left\|e_{j}\right\|_{2}$ is not only bounded by $\left\|e_{j}\right\|_{1}$ but also bounded to rise (if at all) in no pass by more than $0.4 \%$.

A mediocre case: Finally, a zero-phase low-pass filter $\left(2^{\text {nd }}\right.$-order Butterworth, cutoff frequency $5 \mathrm{~Hz}$, see Appendix $\mathrm{C}$ for numerical values) is used as a $\mathrm{Q}$-filter in combination with both of the designed learning gain matrices. Thus, monotonic convergence is no longer provable and convergence of both controllers can only be assessed by simulation for several values of $\left\{n_{j}\right\}$ and $v$. In case of the two-parameter approach this represents a mediocre case since the simple criterion (7) can be used for the optimization of the learning gain matrix, but computationally more expensive simulations are required after Q-Filter design.

\subsection{Simulation results}

The lifted system model $\{P, v, \underline{n}, \bar{n}\}$ from Section 3.1 was simulated with both of the designed controllers $\left\{L_{1}, Q, u_{0}\right\}$ and $\left\{L_{2}, Q, u_{0}\right\}$ for seven passes with pass lengths of $\left\{n_{j}\right\}_{j=0}^{6}=\{34,40,30,46,50,50,37\}$. Simulation results are presented in Figures 1 and 2. In the two-parameter case a core phenomenon of variable pass length learning becomes apparent: Whenever a pass is long enough to encounter samples that have not been reached before, the

8 i.e. $\left(y_{j}\right)_{k}$ shall be below $\left(y_{d}\right)_{k}$ or $\left(y_{0}\right)_{k}$ for all samples $k \in[1,50]$, a practical constraint that avoids too high stimulation intensities 

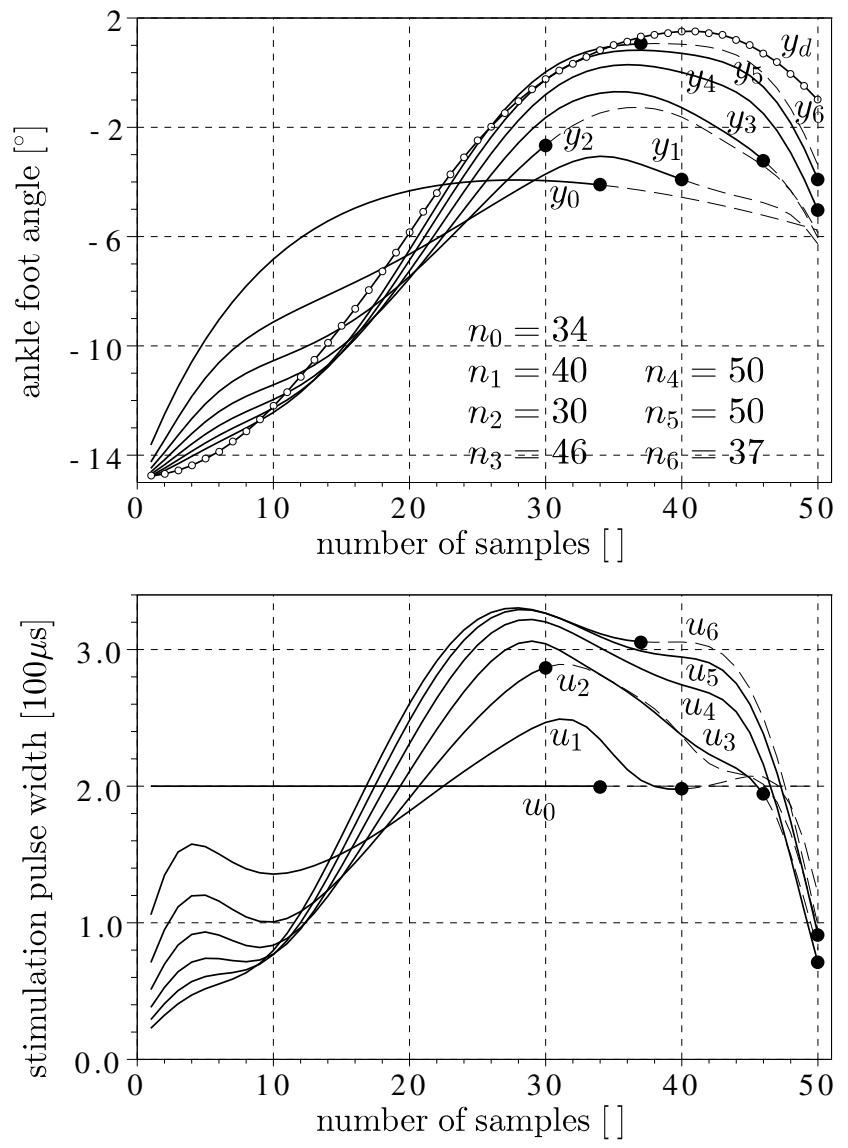

Fig. 1. Variable pass length ILC with diagonal learning gain: Starting from a constant input $u_{0}$ the controller learns to produce the reference $y_{d}$ better and better. The end of each pass is marked with a dot. Lines are continued in dashed style to illustrate the (hypothetical) maximum pass length errors $e_{j}=y_{d}-y_{j}$.

controller starts to learn this piece of trajectory almost from scratch, i.e. starting with the according piece of $y_{0}$. Due to very fast and accurate learning dynamics the very next output already resembles the reference (up to the longest pass length seen so far). In particular the similarity of $y_{2}$ and $y_{3}$ shows that when shorter passes occur, controller performance is not improved significantly any more. In contrast, the diagonal controller learns slower and in a more blurred fashion. But therefore it already roughly approximates the rightmost reference samples when long passes occur for the first time, see e.g. samples $40-46$ of $y_{3}$. Of course, the advantage that lies in this phenomenon might turn against the diagonal approach if either $v$ or $y_{d}$ were changing more rapidly in the region $[\underline{n}, \bar{n}]$. Nevertheless it might be advantageous for some applications and thus it is worth trying to achieve this effect with the faster learning second-(or higher-)order approaches.

\section{CONCLUSION}

The theoretical and yet intuitive concept of the MPL error as the error that the system would undergo if the pass was of full length proved to be useful for convergence analysis of variable pass length ILC systems. The criteria found in Section 2.2 can be used both to check
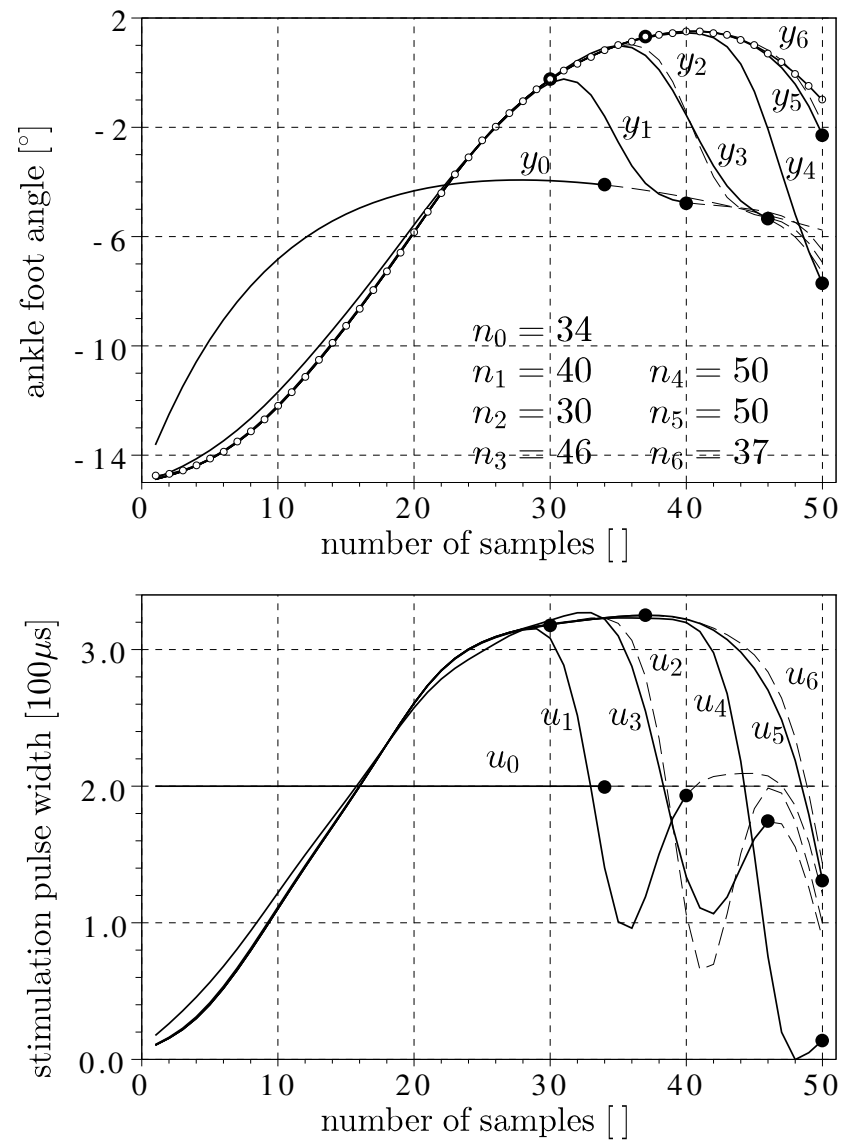

Fig. 2. Variable pass length ILC with two-parameter learning gain: The controller learns to produce the reference (circle markers) clearly faster than the diagonal approach in Figure 1. But the output drops shortly to its initial trace at the end of each pass that is longer than all previous passes, e.g. at the end of $y_{1}$ and $y_{3}$.

monotonic convergence and for controller design itself (as demonstrated in Section 3), even for time-variant systems. Further investigations will include MC conditions for the case of Q-filtering. Research may also aim at simplified criteria for the 2-norm as well as at monotonic convergence of nonlinear variable pass length systems. Moreover, it shall be noted that in the motivational examples in Section 1.2 , as in most other biomedical applications, variable pass lengths are only a subset of the difficulties that complicate the application of ILC theory. Issues like input saturation or iteration-variance (in the process dynamics, the initial conditions, the disturbance, or the reference signal) must be addressed individually as well as in the context of variable pass length systems.

\section{REFERENCES}

[1] D.A. Bristow, M. Tharayil, and A.G. Alleyne. A Survey of Iterative Learning Control. IEEE Control Systems Magazine, 26(3):69-114, 2006.

[2] D.H. Owens and J. Hätönen. Iterative learning control - An optimization paradigm. Annual Reviews in Control, 29:57-70, 2005.

[3] M. Norrlöf and S. Gunnarsson. Time and frequency domain convergence properties in iterative learning control. International Journal of Control, 75(14):1114-1126, 2002. 
[4] K. Galkowski, J. Lam, E. Rogers, S. Xu, B. Sulikowski, W. Paszke and D.H. Owens. LMI based stability analysis and robust controller design for discrete linear repetitive processes. International Journal of Robust and Nonlinear Control, 13:1195-1211, 2003.

[5] Y. Wang, F. Gao, and F.J. Doyle III. Survey on iterative learning control, repetitive control, and runto-run control. Journal of Process Control, 19:15891600, 2009.

[6] H.S. Ahn, Y.Q. Chen, and K.L. Moore. Iterative learning control: brief survey and categorization 1998-2004. IEEE Transactions on Systems, Man, and Cybernetics, Part C: Applications and Reviews, 37(6):1099-1121, 2007.

[7] H. Elci, R.W. Longman, M.Q. Phan, J.N. Juang, and R. Ugoletti. Simple Learning Control Made Practical by Zero-Phase Filtering: Applications to Robotics. IEEE Transactions on Circuits and Systems I: Fundamental Theory and Applications, 49(6):753-767, 2002.

[8] R.W. Longman and K.D. Mombaur. Investigating the Use of Iterative Learning Control and Repetitive Control to Implement Periodic Gaits. Lecture notes in control and information sciences, 340:189-218 , 2006.

[9] H. Nahrstaedt, T. Schauer, R. Shalaby, S. Hesse, J. Raisch. Automatic Control of a Drop-Foot Stimulator Based on Angle Measurement Using Bioimpedance. Artificial Organs, 32(8):649-654, 2008.

[10] H. Nahrstaedt, T. Schauer, S. Hesse, J. Raisch. Iterative Learning Control of a Gait Neuroprosthesis. at - Automatisierungstechnik, 56(9):494-501, 2008.

[11] T. Kailath. Linear Systems, Prentice Hall, 1980.

\section{Appendix A. EXAMPLE OF A LIFTED SYSTEM REPRESENTATION}

Consider the special case of a time-invariant, single-input single-output, state-space system with relative degree one, output disturbance $d \in \mathbb{R}$, and with sampling time $T_{s}$ :

$$
\begin{aligned}
x\left(t+T_{s}\right) & =A x(t)+B u(t), \quad x\left(t_{0}\right)=x_{0} \in \mathbb{R}^{m} \\
y(t) & =C x(t)+d(t)
\end{aligned}
$$

Assume that we want to control the output $y$ in a time interval $t \in\left[t_{0}+T_{s}, t_{0}+n T_{s}\right]$. Then we define the following lifted input and output vectors

$$
\begin{aligned}
& u=\left[u\left(t_{0}\right), u\left(t_{0}+T_{s}\right), \ldots, u\left(t_{0}+(n-1) T_{s}\right)\right]^{T}, \\
& y=\left[y\left(t_{0}+T_{s}\right), y\left(t_{0}+2 T_{s}\right), \ldots, y\left(t_{0}+n T_{s}\right)\right]^{T},
\end{aligned}
$$

and using the Markov parameters ${ }^{9} p_{k}=C A^{k-1} B$ of the above state space system, we find the lifted system representation:

$$
y=\left(\begin{array}{cccc}
p_{1} & 0 & \cdots & 0 \\
p_{2} & p_{1} & \cdots & 0 \\
\vdots & \vdots & \ddots & \vdots \\
p_{n} & p_{n-1} & \cdots & p_{1}
\end{array}\right) u+\left(\begin{array}{c}
C A x_{0}+d\left(t_{0}+T_{s}\right) \\
C A^{2} x_{0}+d\left(t_{0}+2 T_{s}\right) \\
\vdots \\
C A^{n} x_{0}+d\left(t_{0}+n T_{s}\right)
\end{array}\right)
$$

Please note that the zero-input response $v$ (called disturbance signal in Section 1.1 and in most ILC publications) captures both the disturbance $d$ and the initial condition dynamics, and that it is exactly the same signal in each pass. Furthermore, note that the matrix $P$ is Toeplitz and lower triangular, which is not true for time-varying and non-causal systems, respectively.

\section{Appendix B. EXAMPLE OF THE MPL ERROR DYNAMICS MATRICES}

Assume that $\bar{n}=4, \underline{n}=2$, and that

$$
P L=\left(\begin{array}{cccc}
\mathrm{a} & \mathrm{b} & \mathrm{c} & \mathrm{d} \\
\mathrm{e} & \mathrm{f} & \mathrm{g} & \mathrm{h} \\
\mathrm{k} & \mathrm{l} & \mathrm{m} & \mathrm{o} \\
\mathrm{p} & \mathrm{q} & \mathrm{r} & \mathrm{s}
\end{array}\right),
$$

then the MPL error dynamics matrices are given by

$$
\begin{aligned}
\left(I_{\bar{n}}-P L H_{4}\right) & =\left(\begin{array}{llll}
1-\mathrm{a} & -\mathrm{b} & -\mathrm{c} & -\mathrm{d} \\
-\mathrm{e} & 1-\mathrm{f} & -\mathrm{g} & -\mathrm{h} \\
-\mathrm{k} & -\mathrm{l} & 1-\mathrm{m} & -\mathrm{o} \\
-\mathrm{p} & -\mathrm{q} & -\mathrm{r} & 1-\mathrm{s}
\end{array}\right), \\
\left(I_{\bar{n}}-P L H_{3}\right) & =\left(\begin{array}{llll}
1-\mathrm{a} & -\mathrm{b} & -\mathrm{c} & 0 \\
-\mathrm{e} & 1-\mathrm{f} & -\mathrm{g} & 0 \\
-\mathrm{k} & -\mathrm{l} & 1-\mathrm{m} & 0 \\
-\mathrm{p} & -\mathrm{q} & -\mathrm{r} & 1
\end{array}\right), \\
\left(I_{\bar{n}}-P L H_{2}\right) & =\left(\begin{array}{llll}
1-\mathrm{a} & -\mathrm{b} & 0 & 0 \\
-\mathrm{e} & 1-\mathrm{f} & 0 & 0 \\
-\mathrm{k} & -\mathrm{l} & 1 & 0 \\
-\mathrm{p} & -\mathrm{q} & 0 & 1
\end{array}\right) .
\end{aligned}
$$

For perfect plant inversion, $\left(I_{\bar{n}}-\mathrm{PLH}_{4}\right)$ would be a zero matrix, while for $L=0$, the MPL error vector does not change at all.

\section{Appendix C. NUMERICAL VALUES FOR SIMULATION}

The lifted system matrix $P \in \mathbb{R}^{50 \times 50}$ used in Section 3 is Toeplitz and thus completely defined by the values of its first column. These can be gained from the impulse response of (10) by omitting the leading zero-values. Precisely, the first column starts with

$$
P(:, 1)=[0.6634,0.5371551,0.4865000,0.4356716, \ldots] \text {. }
$$

Further, every fifth entry of reference and disturbance, respectively, is provided here (to be connected by splines): $y_{d}(1: 5: 50)=[-14.767,-13.86,-11.679,-8.6404$, $-5.114825,-1.962725,0.0655,1.1659,1.51685,0.71465]$ $v(1: 5: 50)=[-14.952,-14.772,-14.692,-14.712$,

$$
-14.832,-15.052,-15.372,-15.792,-16.312,-16.932]
$$

The learning gain matrix $L_{1}$ is a Toeplitz diagonal matrix with $L_{1}(i, i)=l_{0}=0.12 \forall i \in[1,50]$. The learning gain matrix $L_{2}$ is a Toeplitz matrix with $L_{2}(i, i)=1.5 \forall i \in$ $[1,50]$ and $L_{2}(i+1, i)=-1.35 \forall i \in[1,49]$. All other entries of $L_{2}$ are zero. The Q-filter is calculated in two steps. First a Butterworth filter (cutoff frequency of $5 \mathrm{~Hz}$ ) is designed: $Q(z)=\left(0.067+0.135 z+0.067 z^{2}\right) /\left(0.413-1.143 z+z^{2}\right)$ Then its response to its own time-inverted impulse response gives the first row of its lifted representation $Q(1,:)=[0.214255,0.1898998,0.1331573,0.0738052, \ldots]$. By this vector the symmetric Toeplitz matrix $Q$ is fully defined. It represents a forward-and-then-backward filtering with the above filter $Q(z)$ in the lifted-system domain, and thus attenuates high frequencies without introducing lag. Exploiting the batch nature of ILC, such zero-phase filters are used in many application systems, see e.g. [7].

\footnotetext{
9 i.e. the values of the impulse response, see e.g. [11]
} 